Association of Medical Officers of Asylums and Hospitals for the Insane.

\title{
THE ANNUAL MEETING
}

WILL TAKE PLACE

At LIVERPOOL, on TUESDAY, the 26th of July, 1859 .

Under the Presidency of SIR CHARLES HASTINGS, D.C.L.

THE day of Meeting has been fixed by the Committee, in order to suit the 1 convenience of Members attending the Annnal Meeting of the British Medical Association at Liverpool, on the 27th, 28th, and 29th of July.

The Meeting will be held in the Rooms of the Medical Institution, Mount Pleasant, Liverpool, at 12 noon, precisely. The Meeting will commence with an Address from the President, Sir Charles Rastings, D.c.L. The general business of the Association will afterwards be taken.

The Secretary has received intimation from Dr. Conolly, that several Foreign Psychologists will be proposed by him as Honorary Members.

The Committee invite the attendance of the Profession in Liverpool.

The Dinner will take place at Seven precisely, at Radley's Adelphi Hotel. Members proposing to dine are particularly requested to forward their names to Mr. Radley.

Members of the Profession desirous of admission into the Associntion are requested to communicate with the Honorary Secretary before the day of Meeting.

Notice of communications, and of subjects for diseussion at the Annual Mecting, may be made to the Honorary Secretary.

Members of the Committee are requested to meet at the Medical Institution, at Eleven o'Clock precisely, on the day of Meeting.

The Rules of the Assaciation, as adopted at the Annual Meeting, 1855, cau be had on application to the Honorary Secretary.

C. IOCKHART ROBERTSON, M.B, CANTAB.,

Hayward's Featih, Sussex, 1st July, 1859 Honorary Sectetary.

Just published, fcap. cloth, price 7s. $6 d$.

\section{Brierre de Boismont on Hallucinations.}

A Bistory and Explanation of Apparitions, Visions, Dreams, Eestacy, Magnetism, and Somnambulism. Translated from the French, by RoBERT T. HuLme, F.L.S., M.R.C.S.

LoNDox : Hexkx Rexshaw, 350, Strand.

Just published, 8vo., cloth, price 7s.6d.

The Psychology of Shakespeare. By John Chatres Buckxin, M.D.

Lospon: Losguass AND Co.

Ready in a few days, $8 v 0$.

On the State of Lunacy and of the

Legal Provision for the Insane; with Observations on the Construction and Organization of Asylums. By J. T. Arumbe, M.B., A.B., LOND., L.R.C.P., LoxD, formerly Superintendent of St. Luke's Hospital.

WHLTAM FOLLARD, PRINTER, XONTH STREET, EXETER, 


\section{List of Members-continued.}

LAwıor, Dr., M.S. Killarney Distr. Asy. Lew1s, H., Esq., Co. Asy., Chester

Lown, DR., Saughton Hall, Edinburgh Lowny, Dr, West Malling, Kent.

Mаска тоsн, Da,, M.S. Royal Asy., Gartnavel, Glasgow.

Mackrvтosн, Dr., Dinsdale Park, Darlington.

Macmuxx, Dr., Dist. Asy., Sligo

Macriget, Dr., Somerset Co. Asy. Wells.

Manconm, Dr, V.P. Roy. Asy., Perth

Mantery, Dr., M.S. Co. Asy., Hants.

Marsaini, W. G., Ese., M.S. Co. Asy. Colney Hatch.

Muvostex, Dr. H., Co. Asy., Essex

Maxwers, Dr., M.S. Asy. for Idiots, Redhill, Surrey.

M'Conlough, Dr. D. M., Co. Asy., Abergavenny.

Meyen, Dr., M.S.Suzrey Co.Asy. Wandsworth

Miriasm, G., Eso.

Mrthar, J. N., Eso., Bethnal Green.

Mrlekr, Dil., V.P.St. Thomas Hos, Exeter

Mosno, Dr. H. Cavendish Square, V.P. St. Lnke's London.

Morison, Srr Alexander, M.D., 30 Elgiu Road, Kensington Park

Morrıs, J., Bsq., Peckham Ho. Asy., Lond.

Murrazad, Dz.,Longdales Asy., Bothwell

Nesnit, Dr., M.S., Hospital for the Insane, Northampton.

Niven, Dr., H.E.I.C.S., Med. Depart., Bombay, late Co. Asy. Essex.

Noble, Dik., Manchester.

Norton, Dr.

Orrver, Dz., M.S. Co. Asy., Shropshire.

PAmmer, Dr., M.S. Co.Asy., Lincolnshire.

Psizx, Dr. E., 39, Arlington Street, Mornington Crescent

Parsey,Dr., M.S. Co.Asy., Warwickshire

Pavt, Dr. J. H., Camberwell House, London.

Peppand, Dr., Bushy Park, Limerick.

Prillips, E. T., Ese., M.S. Co. Asy., Haverford West

Purn, Dr., late V.P. St. Luke's

Pownr, Dr., M.S. District Asy., Cork.

Prichard, Da., Abington Abbey, Northampton.

Raz, J., Ese, Naval Asy., Haslar

Romrssos, Dr., Eldon Sq., Newcastle-onTyne
Romertson, J. G., Esq., Co. Asy., Dev Rogas, Dr., M.S. Londonderry Dist. Asy

Ross, Dr. Axprew, Waterloo, near Ports. mouth.

SANikey, H., Esq., Co. Asy. Oxford

SANKEy, Dr., M.S. Co. Asy., Hanwell, Middlesex.

Szaton, Dn., Halliford House, Sunbury. Middlesex.

Sh apter, Dr., V.P.St.Thomas Hos. Exeter. Sarppard, Dr. E., 10, Hanover Terrace. Regent's Park

Shertock, Dr. M.S. Co. Asy. Worcester.

Sinnaz.d, Dn., Roy. Asy., Edinburgh

SкAв, Dr. M.S. Roy. Asy., Edinburgh

Sмrru, James, Ese., Hadham Palace, Herts.

Smrry, Dr. G. P., Park Place, Leeds

Sмrri, Dr. R. M.S., Co. Asy., Durhum

Smrti, Dr.Jno.,Saugliton Hall,Edinburgh

Strviss, Dn., M.S. St. Luke's, London Stiff, Dr., M.S. Co. Asy., Nettingham. Struwell, G. Esa., Bpsom, Surrey.

StruWELl,Dr., Morcroft House, Uxbridge Sutrmnlavd, Dr., Richmond Terrace, Whitehall, V.P. St. Luke's, London

Srmes, J. G., Esa., M.S. Co. Asy. Dorset. Srmes, J. P., Esa., Co. Asy., Wilts.

Taxsen, Dr., Charlotte St., Bedford Sq. Tate, Dr., Lunatic Asy, Nottingham

Teray, J., Bsq., Baillbrook House, Bath.

Thunvam, Dr., M. S. Co. Asy., Wilts.

Tore, Dr. Daniel. H., York.

Trmeman, F. D., Esq., M.S. Co. Asy. Colney Hatch.

WALker, R., Esa., Co. Asy., Kendal.

Warsa, F. D., Bsq., M.S. Hospital for the Insane, Lincoln.

Warson, J. F., Esq., Heigham Hall, Norwich.

WARWICK, J., Eso

West, Dr., M.S. District Asy., Omagh.

Whire, Dr., V.P. Dist. Asy., Derry

WiLletr, Dr., Wylke House, Brentford.

Williams, Da. CaLeB, York.

Wrltrams, Dz., M.S. Co.Asy., Gloucester. Wrisos, R., Esa., M.S. Northumberland Co. Asslum, Alnwick

Wrurav, F., Bsa., Co. Asy. Gloucester.

Wing, Dr, Longwoods House, Bristol.

Woon, Dn, W., Kensington House.

Wroter, Dr. Asvaew, Coleherne Court, Old Brompton.

\section{Honorary Members.}

Boismovr, Dr. Brinanz de, Paris.

Browne, Dr., Gen. Bd. of Lunacy, Edin.

Brodie, Sir Bexjamix Collins, Bart.

D.c.l., Saville Row.

Gaskelt, S.,Esq., Commisst. in Lunacy.

Hollaxd, Sir Henry, Bart, M.D., 25

Brook Street, Grosvenor Square.
Nugent, Dr., Inspector of Asy., Ireland Peacr, Dr., Langley Hall, Derby.

Stanlex, Hans Sloaxe, Nise., Chair. man of Visiting Magistrates, Hants $\mathrm{Co}$ Asy.

W нгте, Dr., Inspector of Asy., Ireland.

Wukrs, J.,Eso., Commissioner in Lunacy 